\title{
How to Supervise International PhD Students: A Narrative Inquiry Study
}

\author{
Mudassir Hussain ${ }^{1} \&$ Hashim Ali $^{2}$ \\ ${ }^{1}$ School of Education, Huazhong University of Science and Technology, Wuhan 430074, China. \\ ${ }^{2}$ Antai College of Economics and Management (ACEM), Shanghai Jiao Tong University, Shanghai 200240, China. \\ Correspondence: Mudassir Hussain, School of Education, Huazhong University of Science and Technology, Wuhan \\ 430074, China. E-mail: mudassir2017@ hust.edu.cn
}

Received: July 24, 2019

Accepted: August 20, 2019

Online Published: August 22, 2019

doi:10.5430/ijhe.v8n5p143

URL: https://doi.org/10.5430/ijhe.v8n5p143

\begin{abstract}
This narrative inquiry study was undertaken, recruiting 06 successful PhD students in China. The participants were invited and semi-structured interviews were taken one-by-one. The study aimed to explore information about supervisor-supervisee relationship and factors that motivate international $\mathrm{PhD}$ students to enhance their research outcomes in a cross-cultural environment. The qualitative data were coded, using QDA miner lite software. After the formation of initial codes, five major categories were emerged included: empowerment, usefulness, success, interest and caring. Each category represented the respective component of MUSIC model of academic motivation (Jones, 2009). The findings illustrated that International PhD students are satisfied with work and life. The supervisors used effective strategies to motivate international $\mathrm{PhD}$ supervisees to enhance academic outcomes. The study uncovered students' expectations which included: formal meetings, feedback, guidance, and team work. Based on study findings and results, the MUSIC model can be used as supervision strategy. It is a comprehensive model where all of its five components cover the supervisees' expectations.
\end{abstract}

Keywords: MUSIC model, motivation, supervisor, supervisee, academic outcomes

\section{Introduction}

Internationalization of higher education and globalization turned the world into a global village. On one hand, it has very positive implications for development in various sectors of society but on the other hand it caused some significant issues in many sectors including higher education. Due to rapid internationalization of higher education and student mobility, universities are experiencing a very heterogenous population of international students. This phenomenon is a hard area for university professors to control due to a complex class composition. To motivate and enhance the academic outcome of international students is a hot topic for researchers. It is not worthy to complain the demotivation of the students but professors who supervise international students need to instill an effective strategy to counter this issue. Ph.D. supervision is a hard job and it becomes harder when it comes to supervise international students. International student's supervision requires a different approach than that of local students. The supervisor shares culture, language and has a thorough knowledge of supervisee's background of knowledge so accordingly the supervisors construct their expectations and generate a strategy to execute research plan. Whereas international students who are a group of diversified population and have even differences among international students within the group; different in education background, different in culture, incompetent in host country's language and so on... These are factors which have been studied deeply in previous research studies. International students are under the pressure of adjustment and acculturative stress apart from adapting academically to unfamiliar education system. Therefore, supervisors of international students should be mindful to all these issues which hinder academic outcomes of international supervisees. By employing the right strategy, supervisors can motivate these students to face all these challenges and fulfill their desired academic outcomes. Motivation is vital for learning, and practicing to master the skills. Motivated students pay attention, engage in learning activities and seek help from others when it is needed (Schunk, Pintrich, \& Meecce, 2008).

Motivation is a complex concept that has been studied through many theories. There are many theories which broaden the knowledge of readers about the motivation especially for teachers to motivate their students in class. These theories explain motivation, human needs and their inter-relationship. Furthermore, researchers have constructed motivation models which are more applicable to be researched and comprehended. Models display a very simple and discernible visualized relationship among its variables or constructs. Similarly, MUSIC model of 
academic motivation (Jones, 2009) which is based on social cognitive theoretical framework. The MUSIC is an acronym of its five included components. Model contains (1) M for eMpowerment, (2) U for Usefulness, (3) S for Success, (4) I for Interest, and (5) C for Caring. This MUSIC Model of academic motivation based on five significant components can be used by teachers while structuring their course materials to enhance the motivation level of the students. But this model can also be used as supervision strategy for international Ph.D. students because its components cover a comprehensive range of international students' needs. Certainly, much of the research and theories upon which the model is constructed can also be applied to a wider range of behaviors, such as those demonstrated in athletics and work environments (Jones, 2009). The term "Motivation" plays a significant role in human life, it guarantees greater outcomes and creates a more satisfied and productive environment. International $\mathrm{Ph} . \mathrm{D}$. students who are a vulnerable population because of many reasons including lacking family and friends' supports, study demands, time restraints and so on, require motivation to utilize their potentials in the most effective way. Academic motivation is not important in itself, but it is important because of its significant role in motivation students to engage in activities which further leads them to academic outcomes (Jones, 2009). The MUSIC model can be used to motivate international supervisees at $\mathrm{PhD}$ level in a cross-cultural environment. The authors consider it as it can be a perfect model for supervisors to motivate the supervisees in research and ensure their timely success.

This study aims to explore the supervision strategies used by professors while supervising international Ph.D. students in Chinese Universities. Nilsson (2008) stated that a limited consideration has been paid to problems related to supervising international supervisees. Previous researches lack sufficient literature on the approaches and strategies which should meet to establish a quality relationship between supervisor and supervisee. International students are the most diversified group of students in Chinese Universities because of their different educational backgrounds, cultures and research skills. Most of the international students are scholarship students for a period of 3-4 years. The students have to fulfill their degree criteria i.e. course work, publication and thesis within this specified time. In case of failure, they will be required to extend by their own expenses. During transition process, adaptation to a new academic and socio-cultural environment, many of these students not only successfully fulfill their criteria but also make some significant researches in their respective fields. The role of the supervisors cannot be denied in the success of the students. The study aims to investigate the strategies these supervisors are using and to see if they are using MUSIC components as strategy to motivate these students to work hard and ensure their academic outcomes within the stipulated time of 3-4 years.

\section{Literature Review}

The purpose of education is not only making an individual learn to write and read but it has to provide an individual with certain skills and social values to prepare him to meet the challenges of the ever-changing world. According to John Dewy; "Education is a progression of living through a constant reconstruction of experiences. It is the development of all those capacities in the individual, which will enable him to overcome his environment and fulfill his possibilities". Teaching is not as much as easy career as it is very often considered. It is something tactical which needs a ceaseless concentration on the part of an instructor. It requires content knowledge, strategy and certain techniques to be put into practice for making learning interesting and for creation of an effective academic environment. How to select or choose the appropriate method, depends upon the situation, learners' potentialities and students centering behavior. They are the educational objectives which provide basis for effective teaching method. To shape the personality's overall design, appropriate techniques and pedagogy is to be employed. Change in methodology will bring about a change in behavior of the learners.

International students face multidimensional problems including adjustment to an unfamiliar environment, issues in understanding host culture and lacking family support but often these issues are unnoticed by faculty members (Lee, 2013). According to Nilsson (2007) there is a significant difference of perception regarding supervisor and supervision between local and international supervisees. He suggested that based on differences between international and local supervisees, the studies conducted with local students cannot be generalized to international students. However, supervisory strategies used with international supervisees derive from the literature on domestic counseling students.

Bernard and Goodyear (2004) very clearly defined supervision as "an intervention that is provided by a senior member of a profession to a junior member or members of that same profession." They asserted that supervisors have to assist the supervisees within the same profession to promote the academic outcomes. It is obligatory for the supervisors to enhance their skills to effectively serve their juniors and supervisees. Smith and $\mathrm{Ng}$ (2009) found that ethically it is the professional objective and duty of the programs, which allow to admit international supervisees, that it should clearly identify the specific needs of these students. Supervisors who deal with international 
supervisees should realize the cultural differences, educational backgrounds and transition issue and pay attention while framing their supervisory relationships. International students' expectations from supervisors may varies in its nature and context because of their diverse needs including, psychological, social, financial and academic. Therefore, the concerned supervisors require to clearly state and understand the expectation of the international students (Mittal, M., \& Wieling, E. , 2006). An appropriate way to understand the demands and expectations of the international students, supervisors need to generalize the approaches from the previously conducted researches but they must ensure the generalization method and reliability (Seo, 2011)

Reynolds and Constantine (2007) stated that previous literature specifies international students are a group of population usually experiencing challenges and difficulties in adjustment which tend them at high risk. They lose their primary support like family and friends, facing challenges of adjustment in a strange environment which naturally cause culture shock (Reynolds \& Constantine, 2007). There are many problems which push international students to be a vulnerable population, the more significant among them is the transition problems (Moores, L., \& Popadiuk, N. , 2011). Results from the findings of Moore and Popadiuk (2011) indicate that international students are under a constant pressure of adjustment issues, coming cross new ideas, cultural norms and unfamiliar academic demands make them feel helpless and depressed at a high stage. Therefore, it is supervisor who needs to understand all these issues and should develop supervisory relationships accordingly. Sterner (2009) examined the perception of the supervisees about supervisors' relationship and how it impacts the work stress and satisfaction. The findings of the study reveal that a positive supervisory relationship results in a satisfied workforce with less work stress and more satisfaction among supervisees. Effective relationship between supervisor and supervisee tends to enhance academic outcomes.

Previous literature highlights that supervisees' perceptions about various type of supervision strategies are correlated with the outcomes (Teven, 2007). Among many outcomes which have been identified to impact by supervisor's behavior, supervisees' perception of supervisor credibility (Cole, \& McCroskey, 2003), subordinate motivation (Adams, Schlueter, \& Barge, 1988) and supervisees' satisfaction (Teven, 2007). According to Tevin (2010) supervisors who are precocial in their power execution as strategy, their supervisees are more satisfied and with more productive work environment. Supervisor holds powers to execute his supervision strategies in a hierarchical order. It has very significant implications either in class-room or work environment for both the phenomena are goal oriented. Supervisor can exercise these powers in different ways and in different styles to execute supervisory plans. This maybe prosocial or antisocial style. Literature elaborated that antisocial use of power in supervision relationships has negative implications on the perceptions of supervisees about their supervisors and cause in stress by lowering satisfaction and many other factors which impact learning, outcomes and productivity (Cranmer, \& Goodboy, 2015).

Scientific publication is the widely recognized evaluation tool for $\mathrm{PhD}$ students, it may be a supplementary requirement for degree completion or opted as future career safety. It is one among the valid measures that is generally used internationally to indicate the talents and research productivity of an individual. This is the tool to publicize results of hours of research and enable interaction among academic communities (Casati, F., Giunchiglia, F., \& Marchese, 2006). Due to its valuable use, publications are crucial for researchers (Lawrence, 2003). It bounds the students to do well in publications or it may endanger their academic career (Fanelli, 2009) According to the study findings of Thomas (1996), the growing demands and within the students' competition is more advantageous than it has disadvantageous effects on research students, and quantification of scientific publication as measurable parameter as other evaluation tools. Intension of scientific publication helps researcher in their self-assessment, to schedule their research activities and plan what they need to do to enhance their publications to achieve greater research productivity and incentives. Sometimes, pressure to compete through publication which is a laborious task may affect their health negatively and causes numerous physical and psychological problems. Research studies have confirmed a significant correlation between publications pressure, burnout symptoms and emotional exhaustion (Tijdink, Vergouwen, \& Smulders, 2013). Moreover, it takes considerable long time in accomplishment of researches and then their writing based on scientific academic principles to prove the findings (Tight, 2010), which ultimately result in less time for social activities like time with family and entertainment which disorganize the balance between work and life.

Jones (2009) expounded MUSIC model of academic motivation based on the critical evaluation of motivation related research and theory. The aim of MUSIC model is to facilitate the instructors to design the courses that engage students in learning and motivate students to take part enthusiastically in teaching-learning activities. The focus is to help instructor control the classroom materials and environment, enabling instructors motivate learners pay attention to achieve the objectives of the courses. The usefulness of the model is intentional decision based on five of its 
components which can guide the instructor to execute their study plans. The MUSIC model includes five components: (1) empowerment, (2) usefulness, (3) success, (4) interest, and (5) caring.

Component

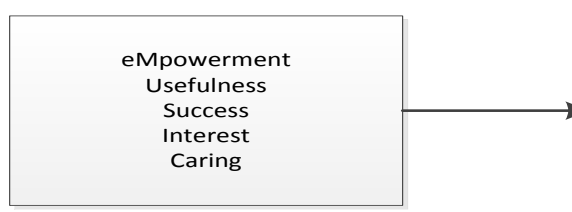

Action

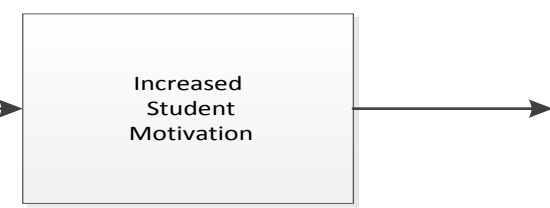

Outcome

ncreased

Student

Learning

Figure 1. A Model based on Social-Cognitive Theoretical Framework, in which Five Component Lead to Increased Students Motivation, Resulting in Increased Student Learning (Jones, 2009)

Academic motivation is significant which motivates students to engage in academic activities that help them achieve their timely success. According to the empowerment component of the MUSIC model, the instructor should allow students to actively choose the course material and be an active participant in decision-making. Instructors should ensure that students understand why the content is useful (Jones, 2009) and to explicitly explain to the material they are learning is related to their interest and it can be beneficial in their future. The instructor can cultivate success in a variety of ways: feedback, assessment, and scheduled assignments can also foster success. Students' interest in their activities is crucial for academic outcomes. Instructors can stir students' interest and stimulate their willingness by designing instruction and coursework that relate much to their backgrounds, level of understanding and have newness, humor, and surprising information. Close interaction, friendly relations, showing concern for success and failure and using cooperative (team work) learning are the few ways that instructors can use for promoting caring. The study aimed to investigate the role of MUSIC components each or all the five as a group by PhD supervisor to know if it can be the model for PhD international students' motivation and result in research productivity and timely success. The study aimed to conduct a narrative inquiry about the lived experiences $06 \mathrm{PhD}$ successful international students to obtain in-depth information about their relationships with supervisors and the role of supervisor in their success to understand the strategies used intentionally or unintentionally administered by their supervisors.

\subsection{Research Questions}

1. How do international Ph.D. students in China narrate their supervisors' supervision strategies?

2. How do supervisor motivate international supervisees to fulfill their degree requirements within funded period of 3-4 years?

3. What are the expectations of international Ph.D. students from their supervisors in China?

\section{Methodology}

The study method was the narrative inquiry. A narrative inquiry is believed to be the real approach of understanding the lived experiences within certain contexts and it contributes to human sense-making (Riessman, 2008). The narrative method assisted the researcher to establish a rapport with participants to learn and exchange the meanings of the stories in the research process that definitely added a validity check to the overall analysis of the study (Creswell, \& Miller, 2000). The site and participants for this study were chosen considering certain criteria like access to the participants and data collection, including validity and ethical concerns (Maxwell, 2005). Huazhong University of Science and Technology (HUST) was chosen as the site for this study. HUST is a famous public sector university, hosting around 3000 international students.

Participants hold the fundamental part of this study; their number and selection criteria are critical to narrative inquiry study. A small number even one or two participants who are accessible and ready to narrate their stories are suitable for narrative inquiry (Creswell, 2007). Therefore, purposeful sampling was used to select settings, participants so that the selections provided unique opportunities for in-depth study (Maxwell, 2005). The sampling was made based on a certain criterion of participants' selection. Creswell (2009) specifies that criterion sampling includes selecting participants who had passed through exact life experiences, suits the narrative study. The main criteria for participant selection were, (a) they should be international students (b) they should be enrolled in their Ph.D. programs and have fulfilled their degree requirements successfully at the time of data collection. This selection provided the researcher with homogeneous background of the participants. The study was performed with a group of 06 Ph.D. international students who fulfilled their degrees in their 3-4 years successfully with distinctions and quality publications. The respondents were all from the same university with different majors, age, gender and nationality. (see Table 1). 
Table 1. Demographic Information of the interview participants

\begin{tabular}{cccccccc}
\hline No & Name & Gender & Age & Major & Nationality & Study duration & Publications \\
\hline $\mathbf{1}$ & P-1 & M & $25-30$ & Engineering & Pakistan & 3 years & SCI 2, NON-SCI 1 \\
$\mathbf{2}$ & P-2 & M & $25-30$ & Engineering & Ethiopia & 4 Years & SCI 1, NON-SCI 2 \\
$\mathbf{3}$ & P-3 & F & $25-30$ & Engineering & Bangladesh & 3 years & SCI 2 \\
$\mathbf{4}$ & P-4 & M & $31-35$ & Engineering & Saudi Arabia & 4 Years & SCI 2, NON- SCI 0 \\
$\mathbf{5}$ & P-5 & F & $25-30$ & Natural Sciences & India & 3 years & SCI 1, NON-SSCI 2 \\
$\mathbf{6}$ & P-6 & F & $25-30$ & Social Sciences & Indonesia & 3 years & SSCI 1
\end{tabular}

A semi-structured interview was used which has been a widely used method of inquiry in narrative studies in social sciences. It allows researcher to take more time with the participants, and obtain in-depth information about the participants' lived experiences (Riessman, 2008). Each interview lasted in approximately 30-40 minutes and the data were carefully noted. For data validity and to avoid missing any information at later stages, the interviews were audio-taped. However, while transcribing the data, no cuts or edits were made to the original responses of the participants and the researcher skillfully added more context to the content to make the responses clear. To ensure a lively discussion with the participants, the interview questions were kept open ended and used a common language of communication (Creswell, 2007)

The interview transcript was coded and further the codes were categorized to manage the themes by using QDA miner light software. Thematic analysis method was used to analyze the interview data. Thematic analysis is a fundamental tool of qualitative data analysis across social sciences which allows qualitative researchers with a flexibility to choose appropriate theoretical framework (Braun, \& Clarke, 2006). Thematic analysis has clarity and flexibility to interpret various aspects of research topic and it further includes guidelines for identifying, analyzing and highlighting the themes (Braun, \& Clarke, 2006).

\section{Results}

Across all the sites 26 generalized topics formed as a result of initial coding process. While coding in focused phase, these codes were clustered into abstract categories. The abstract categories indicated emergent themes from the qualitative data in relation to MUSIC components. An important strength of qualitative data analysis is the ability to identify and model the interactions between several constructs. The participants narrated their stories about their supervisors' strategies for their motivation. Numerous significant factors were described as responsible for their motivation and which enable them to fulfill degree requirements within the designated time of 3-4 year. The most important and frequently used term uttered by participants are given in the words cloud. (see Figure: 2 )

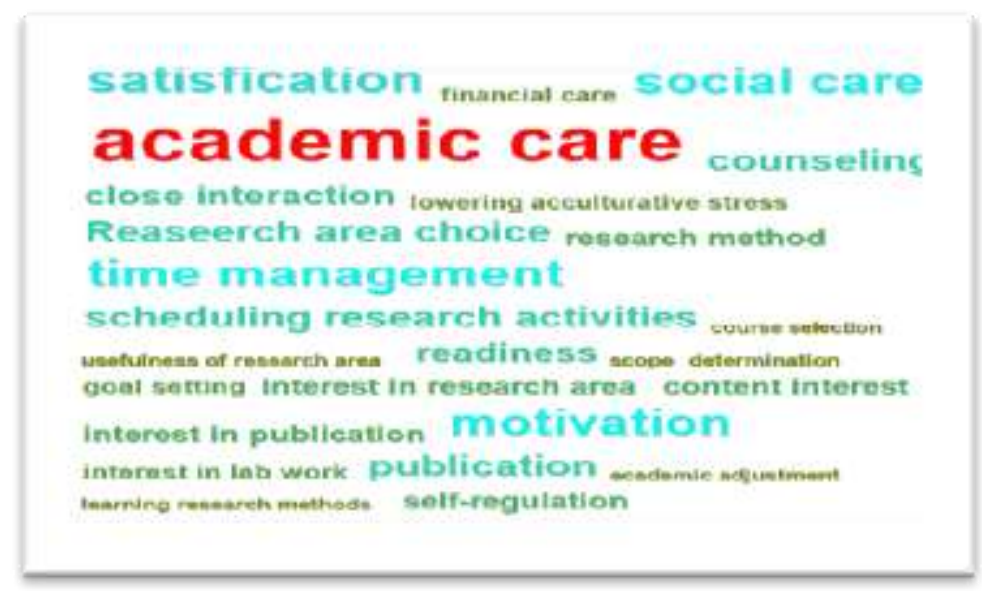

Figure 2. Words cloud, visualizing the frequency of words 
Words cloud showed that the most frequently used term is academic care. This shows that the students stated their satisfaction regarding the academic caring by supervisors. All these can be used as terms to get insight which were parts of supervisors' strategies to motivate these $\mathrm{PhD}$ students and get things done systematically. The frequencies of the word within a script shows the satisfaction or dissatisfaction of the narrators. Similarly, for the validity of the word-count in the words cloud, the code frequency on the basis of cases can be seen as; (see Figure: 3)

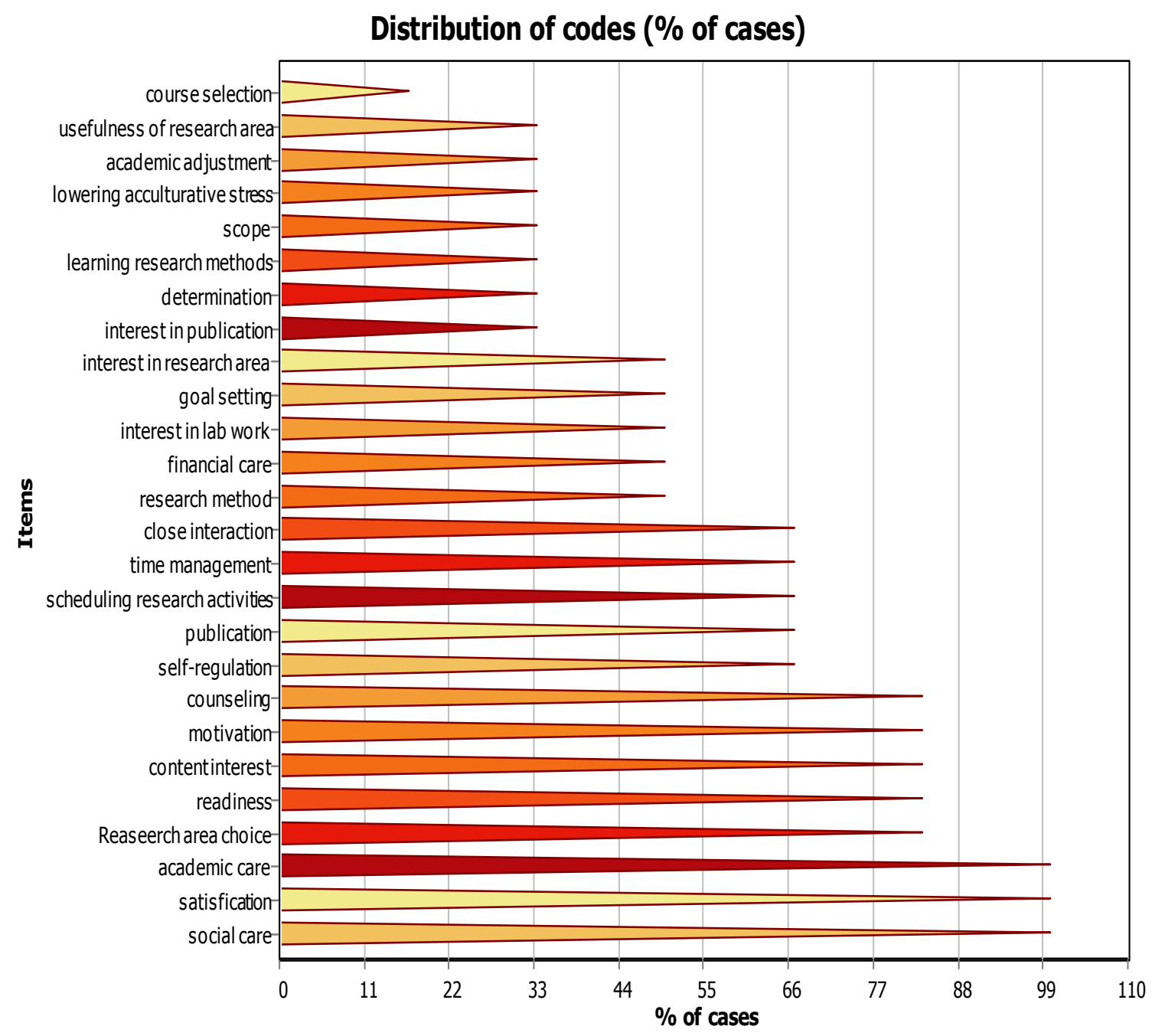

Figure 3. Percentage of cases on each code

Cases refers to participants as case1, case 2, and so on. This study conducted a narrative inquiry targeting 06 participants so this study contains 06 cases altogether. The number of cases on each code represents the vitality of the code. The more cases confirm a certain code, the more it holds the weightage. As in Figure 3, codes; academic care, social care and satisfaction hold the greatest percentage of almost $99 \%$ show that all the 06 respondents showed their agreement to these codes which can be generalized to international $\mathrm{PhD}$ students' perception that their supervisors meet their expectations and they care for them socially and academically. Although there are many factors that can be grouped as codes on later stages of this study. The codes are further visualized and distributed on the basis of codes frequency which showed that which code as a sub-category of generating theme is used more frequently. To understand the frequency of each code emerged during coding process by QDA Miner Lite, see Figure: 4. 


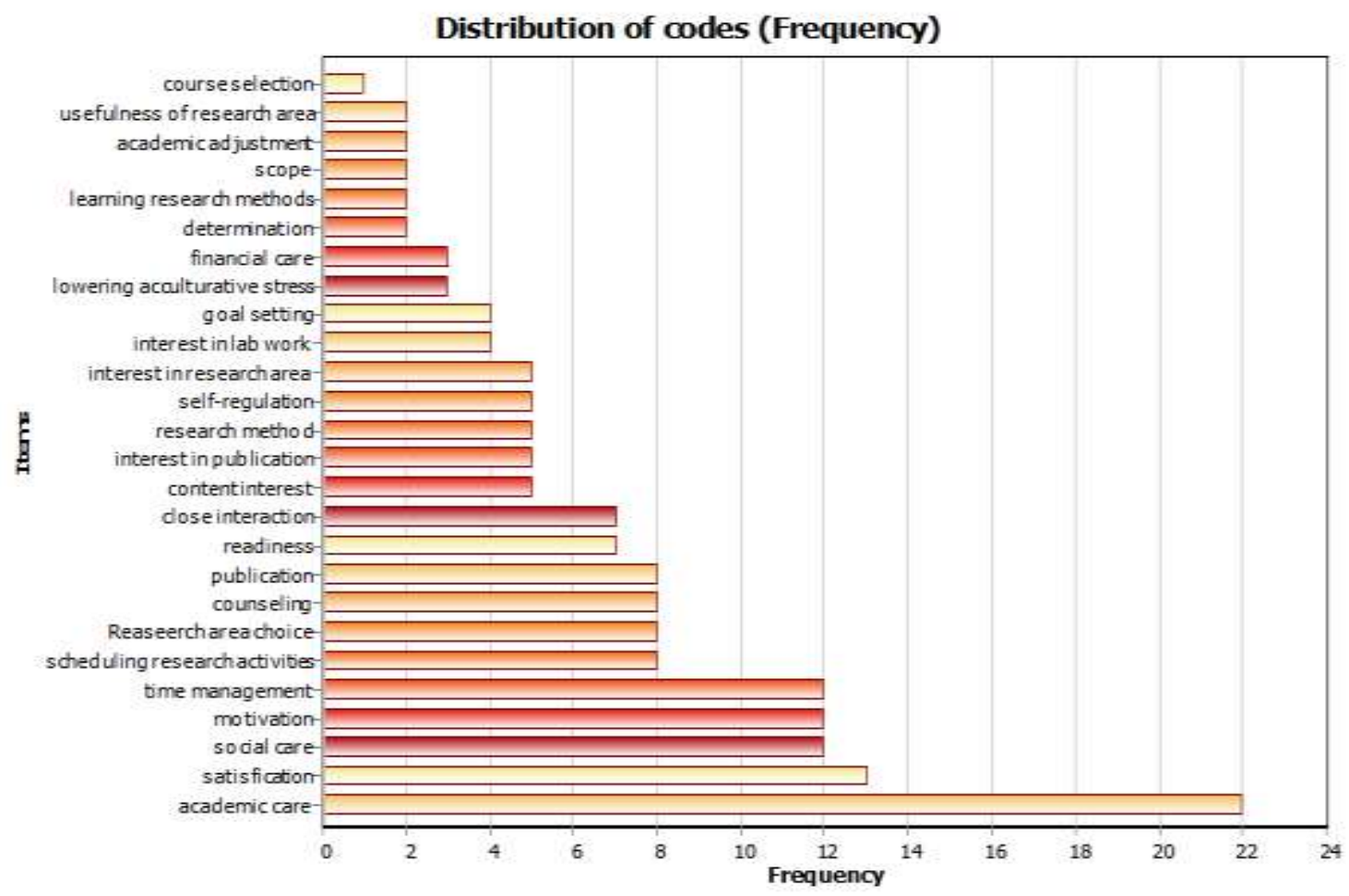

Figure 4. codes' frequency

The overall codes and their respective categories displayed a clear picture of the lived experiences of the participants. In narrative inquiry method, the question arises as to the accuracy of the story looked at objectively even though it must be viewed in a socio-cultural context. The narrative gives one individual to be retrieved on its merit. Such authentication is possible by validation from another narrative. Many researchers use three narratives approach and consider it as appropriate for narrative study while this study conducted semi-structure interviews of six (06) $\mathrm{PhD}$ successful international students to express their experiences and the researcher used this rich data to validate one narrative by another. The cases' frequency on specific codes showed that the collective responses of the maximum cases will further be grouped on basis of count and their respective values.

After coding process, the emerged codes were categorized according to their construction, relation and suitability. It depicted a holistic view of the narratives with a tabular format. The categories which were derived from the MUSIC model were related for proper presentation. (See Table 2) Codes were the representatives of each category which related to the MUSIC model of academic motivation. MUSIC model can be applied to many other disciplines and setting (Jones, 2009) where there is the hierarchical structure of command, the senior requires strategies to motivate juniors or subordinates. In case of supervisor-supervisee relation, research studies illustrated that supervision of international students is quite a different phenomenon as compared to domestic students. Local students are socially and academically adapted group of students with a homogeneous educational background, sharing language, culture and are familiar with the environment whereas international students face many challenges. These challenges are complex and varied in nature, the widely researched are unfamiliarity with the new academic and socio-cultural environment, language barrier, culture shock and lesser support system. The supervisor should realize this valid condition and design a strategy to help student overcome all these challenged and enhance outcome. 
Table 2. codes with their categories

\begin{tabular}{|c|c|c|c|c|c|c|}
\hline Component & Category & Code & Count & $\%$ Codes & Cases & $\%$ Cases \\
\hline & eMpowerment & time management & 12 & $6.60 \%$ & 4 & $66.70 \%$ \\
\hline & eMpowerment & scheduling research activities & 8 & $4.40 \%$ & 4 & $66.70 \%$ \\
\hline \multirow[t]{5}{*}{ M } & eMpowerment & research method & 5 & $2.70 \%$ & 3 & $50.00 \%$ \\
\hline & eMpowerment & course selection & 1 & $0.50 \%$ & 1 & $16.70 \%$ \\
\hline & eMpowerment & research area choice & 8 & $4.40 \%$ & 5 & $83.30 \%$ \\
\hline & Usefulness & usefulness of research area & 2 & $1.10 \%$ & 2 & $33.30 \%$ \\
\hline & Usefulness & Scope & 2 & $1.10 \%$ & 2 & $33.30 \%$ \\
\hline \multirow[t]{4}{*}{$\mathrm{U}$} & Usefulness & readiness & 7 & $3.80 \%$ & 5 & $83.30 \%$ \\
\hline & Usefulness & goal setting & 4 & $2.20 \%$ & 3 & $50.00 \%$ \\
\hline & Usefulness & determination & 2 & $1.10 \%$ & 2 & $33.30 \%$ \\
\hline & Success & self-regulation & 5 & $2.70 \%$ & 4 & $66.70 \%$ \\
\hline \multirow[t]{5}{*}{$\mathrm{S}$} & Success & publication & 8 & $4.40 \%$ & 4 & $66.70 \%$ \\
\hline & Success & learning research methods & 2 & $1.10 \%$ & 2 & $33.30 \%$ \\
\hline & Success & academic adjustment & 2 & $1.10 \%$ & 2 & $33.30 \%$ \\
\hline & Interest & motivation & 12 & $6.60 \%$ & 5 & $83.30 \%$ \\
\hline & Interest & interest in research area & 5 & $2.70 \%$ & 3 & $50.00 \%$ \\
\hline \multirow[t]{6}{*}{ I } & Interest & interest in publication & 5 & $2.70 \%$ & 2 & $33.30 \%$ \\
\hline & Interest & interest in lab work & 4 & $2.20 \%$ & 3 & $50.00 \%$ \\
\hline & Interest & content interest & 5 & $2.70 \%$ & 5 & $83.30 \%$ \\
\hline & Caring & social care & 12 & $6.60 \%$ & 6 & $100.00 \%$ \\
\hline & Caring & satisfaction & 13 & $7.10 \%$ & 6 & $100.00 \%$ \\
\hline & Caring & lowering acculturative stress & 3 & $1.60 \%$ & 2 & $33.30 \%$ \\
\hline \multirow[t]{4}{*}{$\mathrm{C}$} & Caring & financial care & 3 & $1.60 \%$ & 3 & $50.00 \%$ \\
\hline & Caring & counseling & 8 & $4.40 \%$ & 5 & $83.30 \%$ \\
\hline & Caring & close interaction & 7 & $3.80 \%$ & 4 & $66.70 \%$ \\
\hline & Caring & academic care & 22 & $12.10 \%$ & 6 & $100.00 \%$ \\
\hline
\end{tabular}

In Table 2, MUSIC components as categories, subcategories as codes, codes and cases percentages is shown by QDA miner lite software.

In table 2, the results showed that codes describe the categories. The codes count, their percentage, cases and their percentage are shown which illustrate the value of each code. Codes as sub-categories provide the readers with a quick understanding of the phenomenon and they understand the perceptions of the participants. The findings of the study on its respective categories or component confirmed that these codes were the participants' perceptions about their supervisors' attributes and that they played a key role in their success. These attributes portray a picture of supervision strategy or model that is used intentionally or unintentionally but having significant impact on the supervisees' satisfaction, research productivity and academic outcome. Each category which is based on MUSIC 
component may be interpreted in detail to understand the intensity of perception of participants on its respective sub-categories or codes.

\subsection{Results on Each of the MUSIC Component}

\subsection{1 eMpowerment}

eMpowerment is the first component of the MUSIC model of academic motivation. The students should be allowed to play their role in classroom or research related affairs, the student should be the active participant of policy-making process (Jones, 2009). The previous studies findings showed that prosocial supervisors' more positively influence their supervisees as compared to antisocial supervisors and the supervisees enjoy more satisfaction (Teven, 2010). The study findings showed that students described their lived experiences and were found empowered in the various aspect of their academic life which enhanced their satisfaction. Sub-categories of eMpowerment include; research area choice $83.30 \%$, time-management $66.70 \%$, scheduling research activities $66.70 \%$, research method $50.00 \%$, and course selection $16.70 \%$. The percentage shows the number of cases on each code which showed their value and impact respectively. The most international students are empowered, are on research area choice which has a positive impact on their overall satisfaction level as well as it enhances interest in learning and research activities.

\subsubsection{Usefulness}

The usefulness component was extracted from the coded text. The usefulness codes highlighted a significant impact of supervisors on international students in China. The supervisees comprehend the multidimensional usefulness of their research activities; method and materials, lab work and publications during their $\mathrm{PhD}$ study and after $\mathrm{PhD}$ completion for their career. Previous studies illustrated that the students' motivation is influenced by their perception that how the materials they are learning are useful for their future (De Volder, \& Lens, 1082). Researchers have described that the students are more motivated by long term goals as compared to short-term goals (Simons et al., 2004). This study showed that students who were aware of the usefulness of their research area were more satisfied and motivated which resulted in their quality publications, distinctions and successful defense within the time-frame.

For the usefulness category which is originally the second component of MUSIC model of academic motivation, the results demonstrated five codes or sub-categories: readiness $83.30 \%$, goal setting $50.00 \%$, determination $33.30 \%$, scope $33.30 \%$, and usefulness of research area $33.30 \%$. These codes illustrate that supervisors emphasized about the usefulness of the research area and research tools which enhanced the goal setting behavior of supervisees. Students who discern the usefulness of learning material were more motivated and were determined to achieve that goal because the scope of the post-degree scenario was visible to them. It can be concluded that supervisor should explain the usefulness of the material in what the supervisees are engaged in. It will promote determination of the students/supervisees to be focused and ensure academic outcomes.

\subsubsection{Success}

Success is the third important component of MUSIC model of academic motivation. In simple terms, success refers to the mechanism of design which develops students' perception that if they obtain the knowledge and put forth the effort, they can succeed. Learning material should be proportionately easy and challenging for if they are too easy, students will be unmotivated. If they are too difficult and above the level of certain individual or group, students feel themselves helpless and at disadvantage. Therefore, the instructors/supervisors should structure the materials to be challenging, provide feedback about student's knowledge and skills and provide the resources necessary for students to succeed (Jones, 2009). Similarly, literature displayed that international students are a heterogeneous group of students who are diverse in their demographic characteristics as well as in their personal outlooks. The educational system and research culture in their home countries may be different from China, therefore, supervisors should read the background history and accordingly deal with the individual which will enhance the potentials of the students effectively.

The findings of this narrative study demonstrated that success as a category has been branched off into four significant codes which were confirmed by the majority of the participants (cases): publication 66.70\%, self-regulation $66.70 \%$, learning research methods $33.30 \%$, and academic adjustment $33.30 \%$. The supervisor used these coded areas for motivation of international supervisees. Students who were under the time constraint due to financial scholarship were significantly influences by success in all these sub-categories. All the sub-categories are interwoven and have a close link with one another like publications is a success and can be only prompted when a student is academically adjusted and self-regulated. 


\subsubsection{Interest}

Interest is the fourth component of MUSIC model. Interest plays a crucial role in establishing a productive teaching-learning environment and it enhances students learning outcomes. Instructors should develop such instructional materials that could attract the student's attention and engage students in learning activities. A satisfactory engagement of students in an academic set up tend to promote learning outcomes and skill development. Similarly, interest in research area, content knowledge and skills are crucial for accomplishing the goals at PhD level. The supervisor should evolve the strategy to inculcate interest in research activities; lab work, literature review, analysis skills and academic writing. Interest develops a passion among students and to fulfill their academic dreams, they schedule their activities for a systematic approach towards set goals. Interest has very scientifically been defined by Schraw \& Lehman (2001) as it is "liking and willful engagement in a cognitive activity". Interest is a physical state which consists of positive emotions (willingness) and a cognitive component of concentration (the engagement) (Hidi, \& Renninger, 2006).

The findings from the study illustrated that international $\mathrm{PhD}$ students took keen interest in their study and were fully engage in learning and research activities. The participants narrated in detail about the significance of interest in their success and earned academic outcomes. This is the supervisors experience in the field that they boosted supervisees' interest in research by undertaking different strategies. The results illustrated that interest has five sub-categories: motivation $83.30 \%$, content interest $83.30 \%$, interest in research area $50.00 \%$, interest in lab work $50.00 \%$, and interest in publication $33.30 \%$. The results showed that the major sources of their interest in the field is their motivation. The supervisors motivated these students on routine-basis to enhance their interest in research activities and to protect them from depression which is a common in $\mathrm{PhD}$ students' population worldwide. The narratives indicated that international students are motivated and are fully engaged in lab works, learning content knowledge and are eager for scientific publications. The supervisors stirred supervisee's interest in all these dimensions which enhanced the outcomes and ensure international $\mathrm{PhD}$ students within given time period.

\subsubsection{Caring}

Caring is the fifth component of MUSIC model of academic motivation. Caring may refer to different dimensions on the part of a teacher. A caring teacher does not mean that he is a good "buddy" but he must be friendly and meet the academic expectation of students (Jones, 2009). Similarly, supervising PhD students, supervisors need to be friendly and use their authority in a prosocial way. A supervisee upon perceiving the care of supervisor, develops a sense of belongingness, emotional attachment and satisfaction. Humans are social animals and need to establish interpersonal relationships while living in a certain environment and this need becomes more intense when it is the interaction in a cross-cultural environment. International students who belong to different cultural and educational backgrounds need more care as compare to the local $\mathrm{PhD}$ supervisees. International supervisees are required to adjust to the unfamiliar environment which is time taking process and often brings challenges. To cope with these transitional challenges, the significant source is supervisory strategy to care for these vulnerable group of students.

This study results showed that caring has a key role in international students' academic success in China and they enjoyed life satisfaction. The study findings illustrated the caring category is the most significant component which won the more attention of narratives in the study. In-depth analysis of qualitative data further divided the category caring into seven sub-categories/codes: academic care $100.00 \%$, satisfaction $100.00 \%$, social care $100.00 \%$, counseling $83.30 \%$, close interaction $66.70 \%$, financial care $50.00 \%$, and lowering acculturative $33.30 \%$. All the seven sub-categories showed that the supervisors cared on these areas which enhanced their engagement in academic activities. The most significant care which was repeatedly narrated by the participants and loaded on codes, cases and words cloud was academic care (see Fig. 2). Social care includes engaging supervisees with the local students, team work and extra-curricular activities while financial caring refers to the research grants by supervisors. All the participants of this study were scholarship students, funded by Chinese Scholarship Council (CSC), which include tuition fee, lodging, medical insurance and the monthly stipend. The supervisors may provide supervisees with grants for specific research projects and surveys. This comprehensive caring, has very positive impacts on international students' academic outcomes, physical and psychological well beings.

\subsection{Students Expectation}

Previous researches have illustrated that supervisees have certain expectation to meet their academic and social needs. The participants stated in detail about the international $\mathrm{PhD}$ students' expectations, which were based on their lived experiences. The relationship between supervisors and supervisees is a delicate relationship which needs to be overhauled according to the need of the supervisee's expectations and physical conditions. Each international student is different than another in culture, language and educational background. Thus, the expectations of these students 
may also be different up to some extent. After data analysis, on the sub category of students' expectation four sub-categories emerged. These codes showed that international students desire to fulfill their expectations on the mentioned areas which are as under;

Table 3. Students' expectations

\begin{tabular}{clcccc}
\hline \multicolumn{1}{c}{ Category } & \multicolumn{1}{c}{ Code } & Count & \% Codes & Cases & \% Cases \\
\hline Student expectation & feedback & 4 & $2.20 \%$ & 4 & $66.70 \%$ \\
Student expectation & guidance & 3 & $1.60 \%$ & 2 & $33.30 \%$ \\
Student expectation & formal meetings & 5 & $2.70 \%$ & 5 & $83.30 \%$ \\
Student expectation & team work & 3 & $1.60 \%$ & 2 & $33.30 \%$ \\
\hline
\end{tabular}

The findings highlighted students' expectations from their supervisors on the four sub-categories: formal meetings $83.30 \%$, feedback $66.70 \%$, guidance $33.30 \%$, and team work $33.30 \%$. The cases percentage (see Table: 3 ) showed that the international students are concerned on formal meetings and feedback which are very crucial for accomplishing goals. International students expect to have a close interaction with their supervisors and wish to seek frequent guidance on academic issues. These were the expectations which illustrate that close interaction with the supervisor, guidance, feedback and teamwork can help international students put forth all their efforts into action and get excellence in academic achievements.

\section{Discussion}

This narrative inquiry study was undertaken by employing semi-structured interviews to 06 successful $\mathrm{PhD}$ international students at a Huazhong University of Science and Technology, Wuhan P.R. China. The aim of the study was to explore the supervisor-supervisee relationship through the lived experiences of recently graduated international students. The strength of the narrative inquiry method is that it allows the participants to narrate their first hand experiences and the role of the researchers remain a listener. International students in China, were found satisfied and motivated towards their academic obligations. The findings illustrated that the professors who were assigned with the supervision of the international students, perceived the academic and social needs of these students. Thus, satisfied and academically motivated supervisees set their goals and determined to achieve within means and time.

The results stated that supervisors used different strategies to plan the study time, research materials and supervisees' motivation. These "scattered" strategies had a significant impact on the student's academic outcomes: motivation, skill development and publications. Research literature illustrated that international students' expectations were different comparing with local students. Moreover, international students were further varied on different culture, education system and personal outlooks. Thus, a supervisor, unlike class-room activity where a group of students constitute a class, instruct, guide and supervise an individual supervisee. This phenomenon of a direct relation and flow of information between supervisor and supervisee allow supervisor to choose an appropriate strategy to accomplish the task within times and means, like empower students to engage in plans and time-tabling. This will make the students responsible and dedicated.

The study resulted in various codes but finally concluded on five major categories. These five categories are basically the components of MUSIC model. The findings gathered all the emergent codes in a sophisticated way to higher-level categories which confirmed that these components can be used for academic motivation of PhD students and can be an "intentional" model of supervision for supervisors. This will help supervisors understand the real expectations and needs of international students as a whole and as individual as well. Keeping in mind MUSIC model one or two or all of its five components, supervisor can better execute study plan, transmit his ideas and motivate students which result in enhancing learning environment, supervisees' satisfaction and academic outcomes.

The participants of this study were found highly motivated and satisfied. They acknowledged their supervisors and narrated how they supported and motivated them during study period. The qualitative findings illustrated that supervisors were caring which provided satisfaction of work and life. They were found caring in multi-dimensions: academic care, social care, counseling, close interaction, financial care, and lowering acculturative stress. The results showed that supervisors empowered the students in many areas including: research area choice, time management, scheduling research activities, research methods, and course selection. Success is the important component of the model and a significant finding of this study. Researchers stated that students should know that they could get 
through what they were supposed to study. Supervisors properly delivered this component on: publication, self-regulation, learning research methods, and academic adjustment. The participant acknowledged the role of supervisors that they well-defined all the terms, methods and procedure which promoted their skills. According to Jones (Jones, 2009) instructors should design classroom activities in such a manner (interesting) which should attract students' attention and engage them in activity. On interest component, the participants described that their study area, study schedule etc. were appropriate and above all supervisors stirred students' interest by motivation. Interest category included: motivation, content interest, interest in research area, interest in lab work, and interest in publication.

The participants suggested about the expectations of the international PhD students. These suggestions were based on their lived experiences. There were various needs and academic expectations of supervisees from their supervisors but the notable among them were: formal meetings, feedback, guidance, and team work. These expectations were significant among all the cases of qualitative data. International students who were in unfamiliar academic and social environment need time to adjust and often face academic, socio-cultural and psychological problems. While in $\mathrm{PhD}$, their relationship remained with supervisors, so was the basic source of support, guidance and motivation. Meeting, feedback, guidance and team work promote a sense of belongingness, affiliation and satisfaction. It inculcates self-regulation, lowering acculturative stress and enhances academic outcomes.

\section{Conclusion}

The study results displayed three main conclusions which are as follows:

The study was conducted with 06 successful PhDs who recently earned their degrees. The research type was narrative inquiry by employing semi-structured interviews which allowed them freely narrate their stories. Rich data have been gathered and finding showed detailed information. Firstly, international students in China were found satisfied with the supervision strategies, administered by Chinese supervisors. This is because supervision in China is flexible and supervisors deal the students in an appropriate manner. They use different strategies to motivate students. The findings showed that they were caring and they empowered supervisees to take part in managing academic activities. In fact, these strategies have a positive impact on supervisor-supervisee relationship. Secondly, the findings resulted in sophisticated categories of themes which are related to the MUSIC model components. The findings highlighted that the participants confirmed all the five components of the model to be significant for the supervisors to adapt as the supervisor's "intentional" strategy. MUSIC components together can be a comprehensive design for supervisors which cover most of the expectations and academic needs of the international PhD students. Thirdly, the study explained the four important expectations of international students from their supervisors which include: formal meetings, feedback, guidance, and team work. The study suggested that MUSIC model of academic motivation can be used as supervision model for international $\mathrm{PhD}$ students. The model is comprehensive and its components can be used as terms and cannot be confined to one setting as "class-room" This is multi-functional model which can motivate any junior by senior irrespective of environment, work setting or academic level. The authors suggest a further quantitative survey targeting bigger sample to confirm the use of MUSIC model of academic motivation as supervision model for international $\mathrm{PhD}$ students as well as domestic $\mathrm{PhD}$ students and to explore the perception of these students on each of the model's component.

\section{Conflict of Interest}

The authors declare no potential conflict of interest.

\section{References}

Adams, C. H., Schlueter, D. W. \& Barge, J. K. (1988). Communication and motivation within the superior-subordinate dyad: Testing the conventional wisdom of volunteer management. Journal of Applied Communication Research, 16(2), 69.https://doi.org/10.1080/00909888809365274

Bernard, J. M. \& Goodyear, R. K. (2004). Fundamentals of clinical supervision. Needham Heights, MA: Allyn \& Bacon.

Braun, V. \& Clarke, V. (2006). Using thematic analysis in psychology . Qualitative Research in Psychology, 3(2), 77-101. https://doi.org/10.1191/1478088706qp063oa

Casati, F., Giunchiglia \& F. Marchese. (2006). M.Publish and perish: why the current publication and review model is killing research and wasting your money. Retrieved from https://pdfs.semanticscholar.org/778https://doi.org/10.1145/1217821.1226695 
Cole, J. G. \& McCroskey, J. C. (2003). The association of perceived communication apprehension, shyness, and verbal aggression with perceptions of source credibility and affect in organizational and interpersonal contexts. Communication Quarterly, 51(1), 101-110.

Cranmer, G. A. \& Goodboy, A. K. (2015). Power play: Coach power use and athletes' communicative evaluations and responses. Western Journal of Communication, 79, 614-633. https://doi.org/10.1080/10570314.2015.1069389

Creswell, J. (2007). Qualitative inquiry research design: Choosing among five approaches. London: SAGE Publications Inc.

Creswell, J. (2009). Research design: Qualitative, quantitative, and mixed methods approaches (3rd ed.). Thousand Oaks, CA: SAGE Publications, Inc.

Creswell, J. W. \& Miller, D. L. (2000). Determining validity in qualitative inquiry . Theory into Practice, 39(3), 124-131.https://doi.org/10.1207/s15430421tip3903_2

De Volder, M. \& Lens, W. (1082). Academic acheivement and future time perspective as a cognitive-motivational concept . Journal of Personality and Social Psychology, 42(3), 566-571.english.hust.edu.cn/International/Overview.htm. $\quad$ (n.d.). Retrieved from english.hust.edu.cn/International/Overview.htmhttps://doi.org/10.1037/0022-3514.42.3.566

Fanelli, D. (2009). How many scientists fabricate and falsify research? A systematic review and meta-analysis of survey data. PLoS Onehttps://doi.org/10.1371/journal.pone.0005738

Hidi, S. \& Renninger, K. A. (2006). The four-phase model of interest develpoment . Educational Psychologist, 41(2), 111-127.https://doi.org/10.1207/s15326985ep4102_4

Jones, B. D. (2009). Motivation students to engege in learning. International Journal of Teaching and Learning in Higher Education, 21(2), 272-285.

Lawrence, P. A. (2003). The politics of publication. Nature, 422, 259-261.https://doi.org/10.1038/422259a

Lee, K. C. (2013). Training and educating international students in professional psychology: What graduate programs should know. Training and Education in Professional Psychology, 7(1), 61-69.https://doi.org/10.1037/a0031186

Maxwell, J. (2005). Qualitative research design: An interactive approach (2nd ed.). Thousand Oaks, CA: SAGE Publication, Inc.

Mittal, M. \& Wieling, E. (2006). Training experiences of international doctoral students in marriage and family therapy . Journal of Marital and Family Therapy, 369-383.https://doi.org/10.1111/j.1752-0606.2006.tb01613.x

Moores, L. \& Popadiuk, N. (2011). Positive aspects of international student transitions: A qualitative inquiry. Journal of College Student Development, 52(3), 291-306.

Nilsson, J. E. (2007). International students in supervision: Course self-efficacy, stress, and cultural discussions in supervision. The Clinical Supervisor, 26(1-2), 35-47.https://doi.org/10.1300/J001v26n01_04

Nilsson, J. E. \& Wang, C. (2008). Supervising international students in counseling and psychology training. In A. K. Hess, K. D. Hess \& T. H. Hess (Eds.),Psychotherapy supervision: Theory, research, and practice, 70-81. Hoboken, NJ : John Wiley \& Sons.

Reynolds, A. L. \& Constantine, M. G. (2007). Cultural adjustment difficulties and career development of international college students. Journal of Career Assessment,15(3), 338-350.https://doi.org/10.1177/1069072707301218

Riessman, C. K. (2008). Narrative methods for the human sciences. Thousand Oaks, California : Sage.

Schraw, G. \& Lehman, S. (2001). Situational interest: A review of the literature and directions for future research. Educational Psychology Review, 13(1), 23-52.https://doi.org/10.1023/A:1009004801455

Schunk, D. H., Pintrich, P. R. \& Meecce, J. L. (2008). Motivation in education: Theory, research, and applications. Upper Saddle River, NJ: Pearson. 
Seo, Y. S. (2011). Individualism, collectivism, and client expression of different emotions: their relations to perceived counselor effectiveness . Asia Pacific Education Review, 12(2), 251-262.https://doi.org/10.1007/s12564-010-9136-7

Simons, J., Vansteenkinskie, M., Lens, W. \& Lacante, M. (2004). Placing motivation and future time perspective theory in a temporal perspective. Educational Psychological Review, 16(2), 121-139.https://doi.org/10.1023/B:EDPR.0000026609.94841.2f

Smith, S. D. \& Ng, K. (2009). International counseling trainees' experiences and perceptions of their multicultural counseling training in the United States: A mixed method inquiry. International Journal for the Advancement of Counselling,31(4), 271-285.https://doi.org/10.1007/s10447-009-9083-3

Sterner, W. R. (2009). Influence of the supervisory working alliance on supervisee work satisfaction and work-related stress . Journal of Mental Health Counseling, 31(3), 249-263.https://doi.org/10.17744/mehc.31.3.f35441502401831g

Teven, J. J. (2007). Effects of supervisor social influence, nonverbal immediacy, and biological sex on subordinates' perceptions of job satisfaction, liking, and supervisor credibility. Communication Quarterly, 55(2), 155-177. https://doi.org/10.1080/01463370601036036

Teven, J. J. (2010). The effects of supervisor nonverbal immediacy and power use on employees' ratings of credibility and affect for the supervisor. Human Communication, 13(2), 69-85.

Thomas, L. G. (1996). The two faces of competition: dynamic resourcefulness and the hypercompetitive shift. Organization Science, 7(3), 221-242.https://doi.org/10.1287/orsc.7.3.221

Tight, M. (2010). Are academic workloads increasing? The post war survey evidence in the UK. Higher Education Quarterly, 64(2), 200-215.https://doi.org/10.1111/j.1468-2273.2009.00433.x

Tijdink, J. K., Vergouwen, A. C. \& Smulders, Y. M. (2013). Publication pressure and burn out among Dutch medical professors: a nationwide survey. PLoS One, 8, e73381.https://doi.org/10.1371/journal.pone.0073381 